\title{
Operational Dst index prediction model based on combination of artificial neural network and empirical model
}

\author{
Wooyeon Park ${ }^{1,2,3}$, Jaejin Lee ${ }^{3,4, *}$, Kyung-Chan Kim ${ }^{5}$, JongKil Lee ${ }^{3,4}$, Keunchan Park ${ }^{2}$, \\ Yukinaga Miyashita ${ }^{3,4}$, Jongdae Sohn ${ }^{3}$, Jaeheung Park ${ }^{3,4}$, Young-Sil Kwak ${ }^{3,4}$, Junga Hwang ${ }^{3,4}$, \\ Alexander Frias ${ }^{3,6}$, Jiyoung $\mathrm{Kim}^{7}$, and $\mathrm{Yu} \mathrm{Yi}^{2}$ \\ ${ }^{1}$ Korea Meteorological Administration, 61 16-gil Yeouidaebang-ro, Dongjak-gu, Seoul 07062, Republic of Korea \\ 2 Chungnam National University, 99 Daehak-ro, Yuseong-gu, Daejeon 34134, Republic of Korea \\ ${ }^{3}$ Korea Astronomy and Space Science Institute, 776 Daedeok-daero, Yuseong-gu, Daejeon 34055, Republic of Korea \\ ${ }^{4}$ University of Science and Technology, 217, Gajeong-ro, Yuseong-gu, Daejeon 34113, Republic of Korea \\ ${ }^{5}$ Chungbuk National University, 1 Chungdae-ro, Seowon-Gu, Cheongju, Chungbuk 28644, Republic of Korea \\ ${ }^{6}$ York University, 4700 Keele St., Toronto, ON M3J 1P3, Canada \\ ${ }^{7}$ National Meteorological Satellite Centre, KMA, 64-18, Guam-gil, Gwanghyewon-myeonl, Jincheon-gun, Chungcheongbuk-do 27803, \\ Republic of Korea
}

Received 12 June 2020 / Accepted 23 May 2021

\begin{abstract}
In this paper, an operational Dst index prediction model is developed by combining empirical and Artificial Neural Network (ANN) models. ANN algorithms are widely used to predict space weather conditions. While they require a large amount of data for machine learning, large-scale geomagnetic storms have not occurred sufficiently for the last 20 years, Advanced Composition Explorer (ACE) and Deep Space Climate Observatory (DSCOVR) mission operation period. Conversely, the empirical models are based on numerical equations derived from human intuition and are therefore applicable to extrapolate for large storms. In this study, we distinguish between Coronal Mass Ejection (CME) driven and Corotating Interaction Region (CIR) driven storms, estimate the minimum Dst values, and derive an equation for describing the recovery phase. The combined Korea Astronomy and Space Science Institute (KASI) Dst Prediction (KDP) model achieved better performance contrasted to ANN model only. This model could be used practically for space weather operation by extending prediction time to $24 \mathrm{~h}$ and updating the model output every hour.
\end{abstract}

Keywords: Space weather model / Dst index prediction / Artificial neural network

\section{Introduction}

Large-scale interplanetary disturbances from the Sun interact with Earth's magnetic field, resulting in severe space weather events, such as geomagnetic storms (Gonzalez et al., 1994; Ohtani et al., 2000; Bhaskar \& Vichare, 2019). The Dst index is a representative index of geomagnetic activity in the space weather community (Sugiura, 1964; Rangarajan, 1989; Daglis et al., 1999; Wanliss \& Showalter, 2006), and there have been numerous attempts to predict the Dst index (Lyons, 1998; Birn et al., 2001; Raeder \& Maynard, 2001; Rastätter et al., 2013; Eastwood et al., 2017). Empirical and artificial neural network (ANN) models have been handled as important methods for predicting the Dst index (Murayama, 1982; Feldstein, 1992; Boyle et al., 1997; Kugblenu et al., 1999; Weigel, 2010). Many

\footnotetext{
*Corresponding author: jjlee@kasi.re.kr
}

previous studies established that the southward component of the interplanetary magnetic field (IMF) presents the main cause of geomagnetic storms (e.g., Burton et al., 1975; Gonzalez et al., 1994; Echer et al., 2005).

Burton et al. (1975) described the Dst index using the following empirical formula:

$$
\begin{gathered}
\frac{\mathrm{dDst}^{*}}{\mathrm{~d} t}=Q(t)-\frac{\mathrm{Dst}^{*}}{\tau}, \\
\mathrm{Dst}^{*}=\mathrm{Dst}-b \sqrt{P_{\mathrm{dyn}}}+c,
\end{gathered}
$$

where Dst* is the Dst index corrected by solar wind dynamic pressure. The term $Q(t)$ is a function that expresses the rate at which the ring current is intensified by the dusk-ward solar wind electric field (represented in the geocentric solar magnetospheric coordinate system); $P_{\text {dyn }}$ is the solar wind dynamic pressure, $\tau$ is the decay time when the ring current weakens by 
$1 / e$, and $b$ and $c$ are coefficients determined by observational data. As $Q(t)$ is determined solely by the solar wind, we can accurately predict the Dst index using these formulas if we can observe or predict solar wind states. Many Dst models have been derived from this model (Lundstedt et al., 2002), and there have been numerous studies based on the above empirical formula (O'Brien \& McPherron, 2000; Wang et al., 2003) since Burton et al. (1975). The model for predicting the Dst index $1 \mathrm{~h}$ ahead has been significantly improved by Temerin \& Li (2002, 2006).

In the 1990s, advances in computer technology led to ANN for predicting the Dst index (Freeman \& Nagai, 1993; Lundstedt \& Wintoft, 1994; Watanabe et al., 2002). These studies, which used solar wind data as input, initially utilized a simple feedforward ANN. Wu \& Lundstedt (1996) applied a more complex Elman recurrent neural network. More data from solar wind observatories, Wind, and Advanced Composition Explorer (ACE), have been archived and used to improve the results obtained from neural networks. Currently, we can predict the Dst index $1 \mathrm{~h}$ ahead with relatively high accuracy. However, it is not clear whether this is sufficient for space weather operation, as it is not easy to determine the forecast time required for space weather applications. Obviously, more than $1 \mathrm{~h}$ of forecast time is required for space weather applications, changing scheduled satellite operations and the air routes in a polar region, and sending a warning signal to a complex communication system.

As Earth's magnetic field is disturbed almost simultaneously with solar wind variations, it is not easy to predict long-term changes in Earth's magnetic field using only the solar wind data observed at Lagrange point, L1, without forecasting solar winds. Lazzús et al. (2017) created a model that combined ANN with particle swarm optimization and predicted the Dst index 1-6 h ahead. Gruet et al. (2018) combined a long short-term memory recurrent neural network with a Gaussian process to predict the Dst index up to $6 \mathrm{~h}$ ahead. Naturally, the prediction accuracy tends to decrease sharply as prediction time increases. In this study, we also tried to extend the leading time of prediction up to $24 \mathrm{~h}$ differently.

The ACE mission, launched in 1997 and replaced by the Deep Space Climate (DSCOVR) satellite in 2015 for real-time operations, collected considerable solar wind data that can be used by ANN algorithms to predict the Dst index. Nevertheless, it is doubtful whether sufficient data is available for artificial intelligence and machine learning. Since geomagnetic storms with the Dst index of less than $-100 \mathrm{nT}$ occur only a few times a year during the solar maximum and significantly fewer times during the solar minimum, more than 20 years of solar wind data appear to be insufficient for predicting geomagnetic storms (Watari, 2017; Makarov, 2018).

This paper presents three Dst prediction models, simple ANN, empirical model, and combination model (Korea Astronomy and Space Science Institute (KASI) Dst Prediction, KDP). The simple ANN is the baseline model for comparison. Here, we just tried to follow the existing neural network algorithm, so there are no significant advances and show a comparable prediction performance to the previous models, such as shown in Section 3. The empirical model (equations) indicates the intensity of a magnetic storm according to the degree of solar wind changes. Two kinds of geomagnetic storms are considered separately; coronal mass ejection (CME) driven and corotating interaction region (CIR) driven storms in Section 4. This preemptive information can help improve Dst prediction accuracy because a space weather operator can determine in advance whether CIR or CME causes future geomagnetic storms. Finally, the present study proposes a combination of empirical and ANN models in Section 5. The predicted values by the empirical model are used as an input parameter to the ANN model. Thus we could improve the prediction performance better than other models.

The method combining empirical and ANN models is similar to that of Revallo et al. $(2014,2015)$; the difference is in the empirical model. They used time histories of solar wind and magnetosphere interaction proposed by Romashets et al. (2008) a neural network input data. They constructed an analytical representation of magnetic fields in the region where the solar wind interacted with the earth's magnetosphere and used the results as an input parameter for predicting the Dst index. On the other hand, we directly derived the Dst index's prediction values from the empirical equations and applied them to the ANN model. Thus, the KDP model could extend the prediction time up to $24 \mathrm{~h}$ while Revallo et al. $(2014,2015)$ predict the Dst index just $1 \mathrm{~h}$ ahead. See and compare the model performance in Figure 9.

\section{Data}

For the development of a model, we use the solar wind data collected by the ACE and DSCOVR satellite and the Dst index as input values for the prediction model from 1999 to 2017 . The Dst index is obtained from NASA (https://omniweb.gsfc.nasa. gov/ow.html), and the hourly average solar wind data are acquired from CDAWeb (https://cdaweb.gsfc.nasa.gov/index. html). For the model's operation, current Dst and solar wind data are downloaded from World Data Center for Geomagnetism, Kyoto (http://wdc.kugi.kyoto-u.ac.jp/dst_realtime/presentmonth/ index.html) and NOAA (https://services.swpc.noaa.gov/products/ solar-wind/).

Tables A.1 and A.2 in Appendix list the CME- and CIRdriven geomagnetic storms from February 1999 to September 2017. Here, the geomagnetic storm is defined with a minimum Dst index of less than $-50 \mathrm{nT}$. The event start time is defined as the maximum Dst time at the main phase, and the event end time is the recovery time exceeds $-30 \mathrm{nT}$. These storm lists are mainly obtained from published papers (referenced therein), and some CIR-driven storms are added in this study. The effects of CME and CIR are known to be different (Liemohn et al., 2010). CME can potentially generate large geomagnetic storms, while CIR generally triggers minor storms. The empirical model developed in this study distinguishes the CME and CIR-driven storms, and the empirical model results are used as the input parameter of the combination model.

\section{Artificial neural network (ANN) model}

This section introduces a simple ANN model and shows its performance. The model is combined with an empirical model in Section 5. The ANN used in this study is the feed-forward network (Gardner \& Dorling, 1998) known as the most simple 


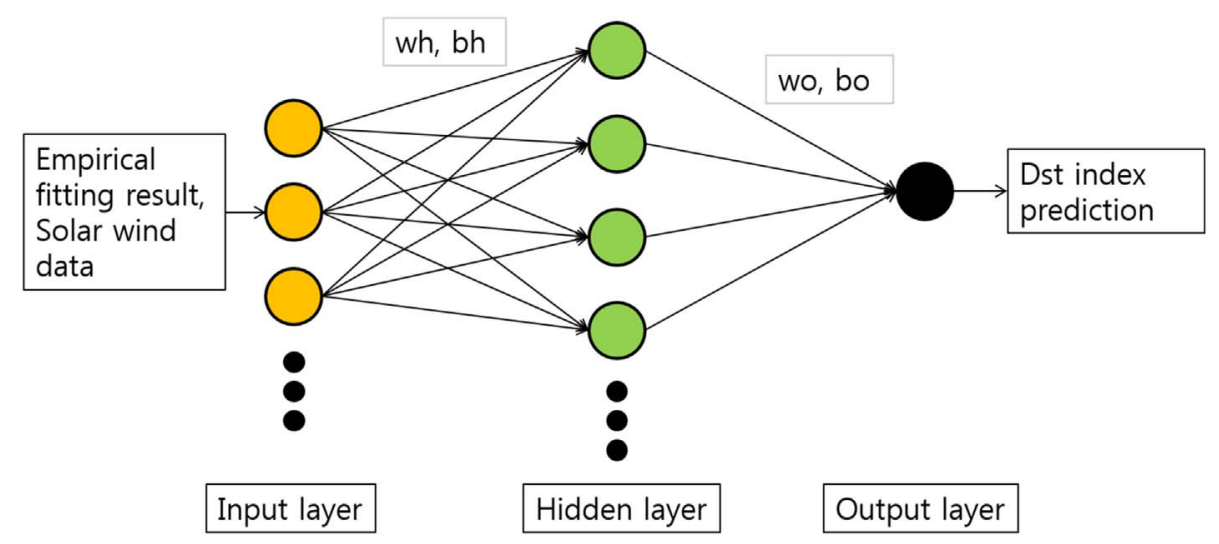

Fig. 1. Artificial neural network architecture consisting of input, hidden, and output layers.

Table 1. Simple artificial neural network input parameters.

\begin{tabular}{ll}
\hline Parameter & Description \\
\hline $\mathrm{N}(\mathrm{tc})$ & Current solar wind density \\
$\mathrm{N}(\mathrm{tc})-\mathrm{N}(\mathrm{tc}-\mathrm{1})$ & Difference between current solar wind density and value obtained 1 $\mathrm{h}$ earlier \\
$\mathrm{V}(\mathrm{tc})$ & Current solar wind speed \\
$\mathrm{V}(\mathrm{tc})-\mathrm{V}(\mathrm{tc}-1)$ & Difference between current solar wind speed and value obtained $1 \mathrm{~h}$ earlier \\
$\mathrm{Bt}$ & Current total IMF \\
$\mathrm{Bt}(\mathrm{tc})-\mathrm{Bt}(\mathrm{tc}-1)$ & Difference between current total IMF and value obtained $1 \mathrm{~h}$ earlier \\
$\mathrm{Bz}(\mathrm{tc})$ & Current IMF Bz \\
$\mathrm{Bz}(\mathrm{tc}-1)$ & IMF Bz obtained $1 \mathrm{~h}$ earlier \\
$\mathrm{Bz}(\mathrm{tc}-2)$ & IMF Bz obtained $2 \mathrm{~h}$ earlier \\
$\mathrm{Bz}(\mathrm{tc})-\mathrm{Bz}(\mathrm{tc}-1)$ & Difference between current IMF Bz and value obtained $1 \mathrm{~h}$ earlier \\
$\mathrm{Bz}(\mathrm{tc}-1)-\mathrm{Bz}(\mathrm{tc}-2)$ & Difference between IMF Bz values obtained one and $2 \mathrm{~h}$ earlier \\
$\mathrm{Dst}(\mathrm{tc})$ & Current measured Dst index \\
$\mathrm{Dst}(\mathrm{tc}-1)$ & Measured Dst index values obtained $1 \mathrm{~h}$ earlier \\
$\mathrm{Dst}(\mathrm{tc}-2)$ & Measured Dst index values obtained 2 $\mathrm{h}$ earlier \\
\hline
\end{tabular}

ANN-type algorithm (Haykin, 1998). The training technique of weights and biases uses an error backpropagation learning algorithm with a gradient descent learning method. The basic structure of this ANN is composed of an input layer $(I)$, a hidden layer $(H)$, and an output layer $(O)$ as shown in Figure 1. The hidden layer uses a nonlinear hyperbolic tangent function, $H=\tanh (I)$, and the output layer uses the $O=H$ type linear function. The following equations express these layers:

$$
\begin{gathered}
H_{j}=\tanh \left(\sum_{i=1}^{N} w h_{i, j}+b_{i}\right), \\
O_{k}=\sum_{j=1}^{M} w o_{j, k} H_{j}+b_{k},
\end{gathered}
$$

where $H_{j}$ and $O_{k}$ are the nodes of the hidden and output layers, respectively, $w h_{i, j}, w o_{j, k}$ are the weights of the hidden and output layers, and $b_{i}$ and $b_{k}$ are biases, respectively. The suffixes $i, j$, and $k$ denote the node numbers in the input, hidden, and output layers, and $N$ and $M$ denote the total number of nodes in the input and hidden layers, respectively.

We use the input parameters listed in Table 1 for the input layer. Note that the simple ANN model uses the data collected for storm and quiet time and does not distinguish CME and CIR-driven storms. We use the current observations, and the differences between the current observations and the observations obtained 1 or $2 \mathrm{~h}$ earlier. The use of these differences as input data helps predict the Dst index trend and, therefore, plays an important role in predicting the Dst value more than $1 \mathrm{~h}$ ahead. The differences are estimated based on hourly averaged solar wind data. We also predict 1-24 h later Dst index as the target values of the output layer. The output layer has one node, as shown in Figure 1, which means the simple ANN model consists of 24 models for each hour predictions in advance.

We train the model by adjusting hyperparameters such as the node numbers of the input and hidden layers, learning rate, and learning cycle to optimize the neural network. The optimization selects the best condition by calculating the correlation coefficient and root mean square error (RMSE) between the predicted and actual values for all hyperparameters. The equations are given as follows:

$$
\begin{gathered}
\mathrm{CC}=\frac{\sum_{i=1}^{N}\left(Y_{\text {pre }} Y_{\text {real }}\right)_{i}-N \bar{Y}_{\text {pre }} \bar{Y}_{\text {real }}}{\sqrt{\left(\sum_{i=1}^{N}\left(Y_{\text {pre }}^{2}\right)_{i}-N \bar{Y}_{\text {pre }}^{2}\right)} \sqrt{\left(\sum_{i=1}^{N}\left(Y_{\text {real }}^{2}\right)_{i}-N \bar{Y}_{\text {real }}^{2}\right)}}, \\
\mathrm{RMSE}=\sqrt{\frac{\sum_{i=1}^{N}\left(Y_{\text {pre }}-Y_{\text {real }}\right)_{i}^{2}}{N},}
\end{gathered}
$$

where $Y_{\text {pre }}$ is a predicted value, $Y_{\text {real }}$ is a measured value, and $N$ is the number of values. 
Table 2. Optimized hyperparameters of ANN models

\begin{tabular}{lccc}
\hline & $\begin{array}{c}\text { Hidden layer } \\
\text { node number }\end{array}$ & $\begin{array}{c}\text { Learning } \\
\text { rate }\end{array}$ & $\begin{array}{c}\text { Learning } \\
\text { cycle }\end{array}$ \\
\hline 1-hour prediction & 13 & 0.0008 & 1500 \\
2-hour prediction & 50 & 0.001 & 1400 \\
3-hour prediction & 7 & 0.01 & 1500 \\
4-hour prediction & 41 & 0.001 & 1500 \\
5-hour prediction & 30 & 0.001 & 1300 \\
6-hour prediction & 45 & 0.001 & 1200 \\
7-hour prediction & 40 & 0.0001 & 1400 \\
8-hour prediction & 25 & 0.0001 & 1200 \\
9-hour prediction & 25 & 0.0001 & 1200 \\
10-hour prediction & 25 & 0.0001 & 1200 \\
11-hour prediction & 25 & 0.0001 & 1200 \\
12-hour prediction & 25 & 0.0001 & 1200 \\
13-hour prediction & 25 & 0.0001 & 1200 \\
14-hour prediction & 10 & 0.0001 & 1300 \\
15-hour prediction & 11 & 0.0001 & 1300 \\
16-hour prediction & 11 & 0.0001 & 1300 \\
17-hour prediction & 11 & 0.0001 & 1300 \\
18-hour prediction & 11 & 0.0001 & 1300 \\
19-hour prediction & 11 & 0.0001 & 1300 \\
20-hour prediction & 11 & 0.0001 & 1300 \\
21-hour prediction & 11 & 0.0001 & 1300 \\
22-hour prediction & 11 & 0.0001 & 1300 \\
23-hour prediction & 11 & 0.0001 & 1300 \\
24-hour prediction & 11 & 0.0001 & 1300 \\
\hline
\end{tabular}

For performance optimization, all hidden layers with node numbers of 7-50, a learning rate of $0.0001-0.01$, and learning cycles of 1200-1500 were trained and validated (see Table 2). For the ANN training, we used the data observed in 1999, 2001, 2004, 2007, 2008, 2011, 2012, 2014, 2016, and 2017. For the model validation, we use 2000, 2002, 2006, and 2013, and for the test, we use 2003, 2005, 2009, 2015. A total of 53,839, 28,526, and 25,421 data points are used for training, validation, and testing, respectively. The data covered storm and quiet times.

The red dots (ANN model) in Figure 2 show the RMSE and the correlation coefficients obtained by comparing the prediction results with the observed Dst index during the test period. The statistical analysis is conducted for the entire period without dividing it into the storm and quiet period regardless of distinguishing between CME- and CIR-driven geomagnetic storms. Compared with other models, this figure also shows the results from the various ANN models reported in the literature (Lazzús et al., 2017). As seen in Figure 2, many models predict the Dst index just $1 \mathrm{~h}$ ahead, and more advanced models (Wu \& Lundstedt, 1997; Stepanova et al., 2005) can predict up to $6 \mathrm{~h}$, while our model attempts to predict the Dst index up to $24 \mathrm{~h}$. The initial version of the ANN shows similar or slightly lower performance than other models because no improved techniques are used.

\section{Empirical model}

The relationship between the solar wind and the Dst index could be derived using an arbitrary empirical formula (McPherron \& O'Brien, 2001). In deriving the equations, we
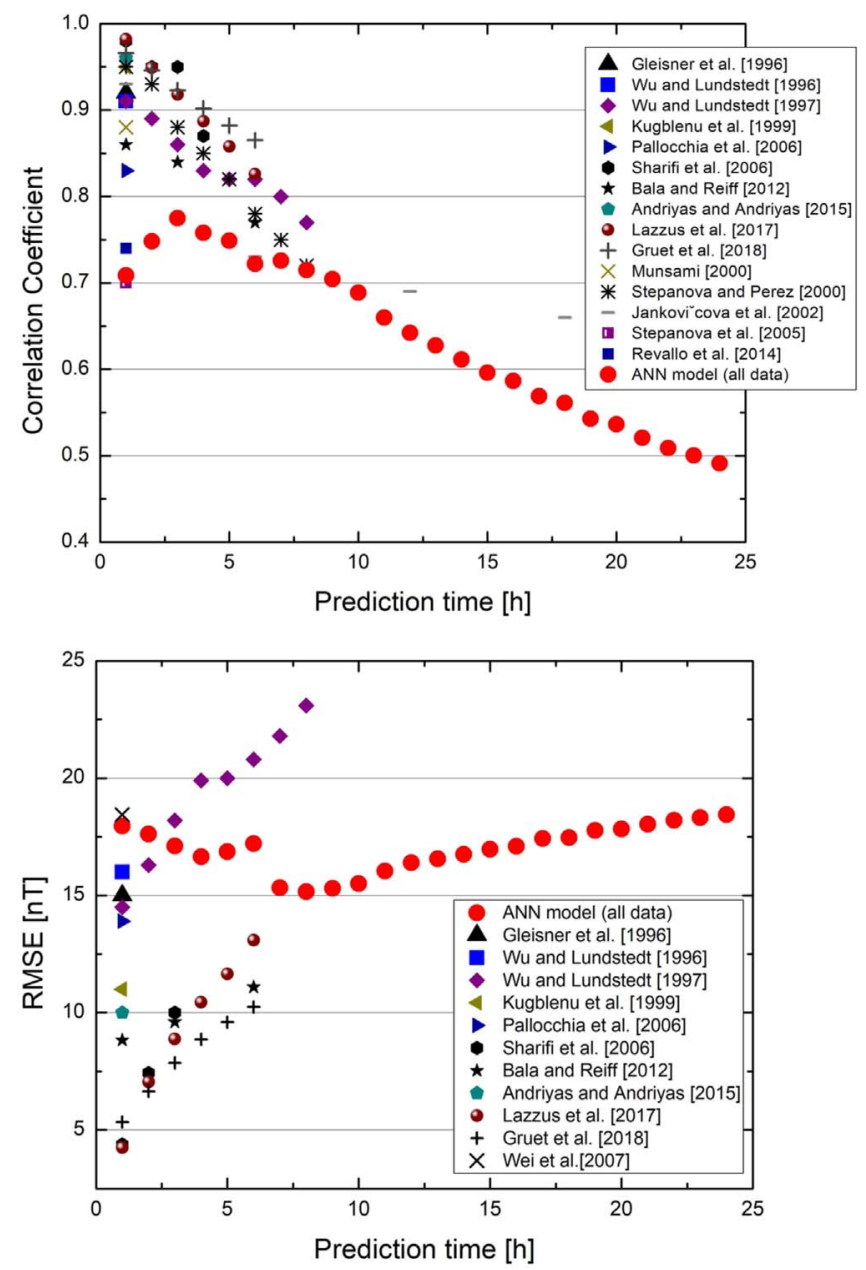

Fig. 2. Comparison of the simple ANN model performance with other models: correlation coefficient (up) and RMSE (down) derived for test period.

regarded a geomagnetic storm as having only one main phase and one recovery phase to simplify the relationship between the measured Dst index and input parameters. However, this model could also be used to predict the multi-peak storms when combined with the ANN model. The empirical model consists of three equations: predicting the minimum Dst index, finding the time reaching Dst Minimum, and expressing the recovery phases.

$$
\begin{aligned}
\mathrm{Dst}_{\text {min }}(\mathrm{CME})= & 0.36 \times(V \times B s \times 0.001)^{2} \times\left(\frac{N}{10}\right) \times \sqrt{\frac{B s}{7}} \\
& \times e^{\frac{\mid \text { Dstcurrent }^{100}}{100}}+\sqrt{0.000317 \times N \times V^{2}} \\
\mathrm{Dst}_{\text {min }}(\mathrm{CIR})= & 5.24 \times(V \times B s \times 0.001+1) \times \sqrt{\frac{B S}{9}} \\
& \times e^{\frac{\mid \text { Dstcurrent } \mid}{450}}+\sqrt{0.000156 N \times V^{2}} .
\end{aligned}
$$

Equations (7) and (8) are used to determine the minimum Dst index $\left(\mathrm{Dst}_{\min }\right)$ from the current solar wind condition caused 

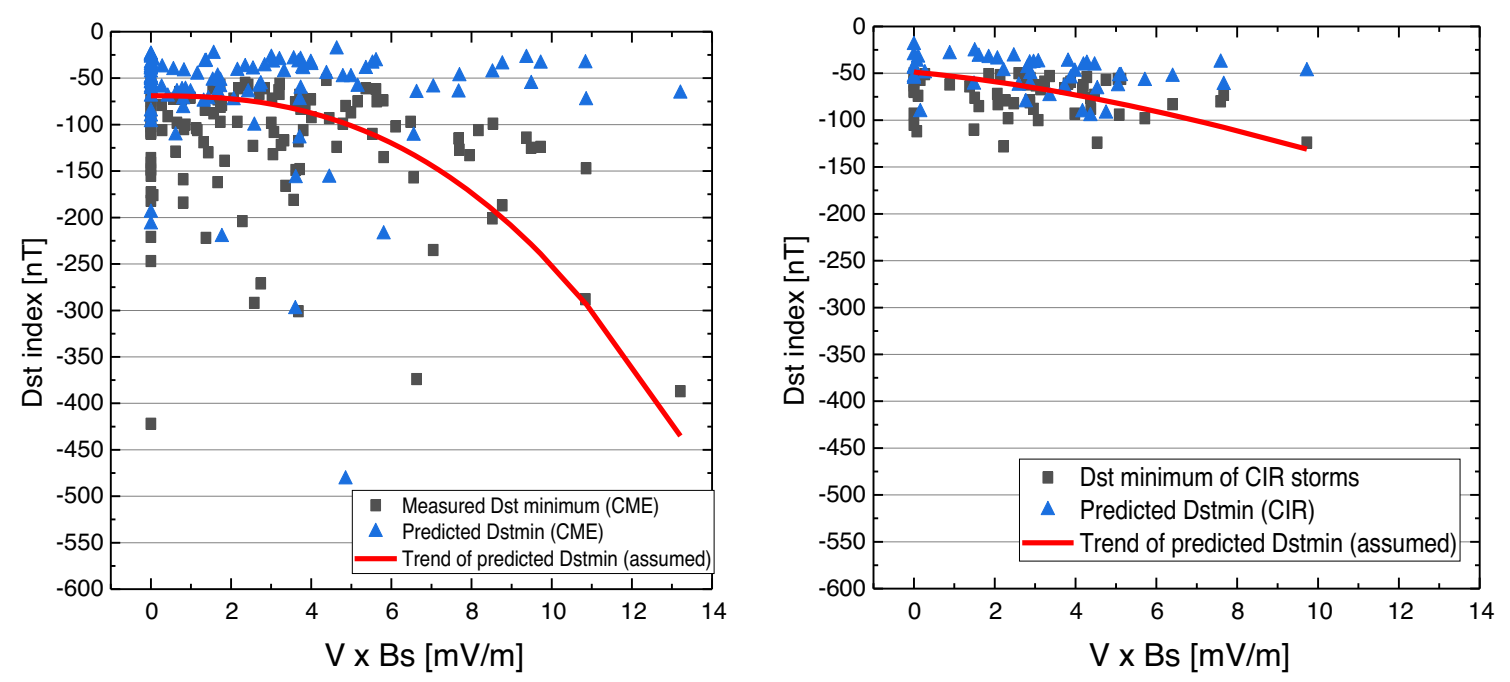

Fig. 3. Predicted minimum Dst (blue) and corresponding measured minimum Dst (black) with respect to $V \times B s$ for CME-driven (left) and CIR-driven (right) storms. The red lines show the values calculated from equations (7) and (8) with $B t=B s, V=500 \mathrm{~km} / \mathrm{s}$, and $N=30 / \mathrm{cm}^{3}$.

by CME and CIR, respectively. While Burton et al. (1975) expressed the ring current injection rate in terms of the solar wind speed $(V)$ and the southward IMF $(B s)$, in this work, we add the solar wind density $(N)$, total IMF $(B t)$, and current Dst index to complete the equation. The solar wind parameters on the right-hand side of equations (7) and (8) are measurements when the model starts to run each hour. Recall that this model is designed to produce new prediction values every hour for the space weather operation. These equations were derived by a polynomial fitting algorithm in commercial software for the storm period and added exponential terms for adjusting large storm trends. In deriving these equations, we fixed some arbitrary coefficients and fit a function to the measurements to find the unfixed coefficients. We do not think we found the optimum values describing minimum Dst because the equations have six input parameters. Note that there is still a large inconsistency between prediction and measurements in Figure 3. Nevertheless, these empirical equations effectively build the combination model, as shown in Section 5.

At the initial storm phase, the predicted Dst ${ }_{\text {min }}$ is mainly controlled by solar wind measurements because Dst current $_{\text {is }}$ generally a small value, and the term, $e^{\frac{\mid \text { Dstcurent }}{450}}$ does not significantly contribute to the Dst ${ }_{\text {min }}$ prediction. However, as a big storm progresses, only solar wind data are not enough to describe geomagnetic storm developments. The exponential term adjusts the estimation of $\mathrm{Dst}_{\text {min }}$. Figure 3 shows the minimum Dst predicted by using equations (7) and (8) (blue symbols), measured minimum Dst (black symbols), and trend (red line) of equations (7) and (8) under specific conditions, $B t=B s, V=500 \mathrm{~km} / \mathrm{s}$, and $N=30 / \mathrm{cm}^{3}$. In these figures, the predicted Dst ${ }_{\text {min }}$ is calculated at the initial phase of the storm when $V \times B s$ is just over $0.49 \mathrm{mV} / \mathrm{m}$, and several hours later, minimum Dst measurement is obtained. As shown in Figure 3, the frequency of smaller geomagnetic storms is higher. For the prediction algorithms based on artificial intelligence, a larger weighting factor is applied to more frequent storms. Therefore, the ANN algorithm shows the worse prediction accuracy for the greater storms. In contrast, the empirical models are derived by human intuition and attempt to find a correlation between minimum Dst index and input parameters, regardless of the amount of data.

Next, we calculate the time $\left(T_{\min }\right)$ required to reach the minimum Dst obtained from equations (7) and (8). If we could estimate the Dst variation (dDst) for $1 \mathrm{~h}$, we can estimate $T_{\min }$ as the following equation:

$$
T_{\min }=\mathrm{Dst}_{\min } / \mathrm{dDst} .
$$

As the Dst index represents the variation in the geomagnetic field due to ring current development, the amount of change in the Dst index is proportional to the injection rate of the ring current particles. In this study, we adopt Wang et al. (2003) empirical model to derive the rate of change in Dst per hour (dDst) during the storm main phase.

$$
\begin{gathered}
\frac{\mathrm{dDst}}{\mathrm{d} t}=Q-\frac{\mathrm{Dst}^{*}}{\tau}, \\
\mathrm{Dst}^{*}=\mathrm{Dst}-7.26 \sqrt{P}+11 \mathrm{nT}, \\
0 \quad \begin{array}{cl}
V B_{z} \leq 0.49 \mathrm{mV} / \mathrm{m} \\
-4.4\left(V B_{z}-0.49\right)\left(\frac{P}{P_{0}}\right)^{\gamma}, & V B_{z} \geq 0.49 \mathrm{mV} / \mathrm{m}
\end{array} \\
\tau=\left\{\begin{array}{cc}
8.70 e^{\frac{6.66}{(6.04+P)}}, & B_{z} \geq 0 \\
2.40 e^{\frac{9.74}{\left(4.69+V B_{z}\right)}}, & B_{z}<0
\end{array}\right.
\end{gathered}
$$

where $P$ (in $\mathrm{nPa}$ ) is the solar wind dynamic pressure and $P_{0}$ and $\gamma$ are parameters provided by Wang et al. (2003). These parameters depend on the Dst index, as shown in Table 3. Here, the Dst index refers to a measurement at the current time.

For more information about the model, refer to Wang et al. (2003). Now, we obtain the dDst, and the prediction equation for the main phase can be expressed as follows:

$$
\operatorname{Dst}(t)_{\text {predict }}=\text { Dst }_{\text {current }}+\left(\frac{\mathrm{dDst}}{\mathrm{d} t} \times t\right) \text { where } t=1 \sim T_{\min }
$$


Table 3. Parameters which used for calculating the ring current injection rate, $Q$.

\begin{tabular}{lcc}
\hline Dst index & $P_{0}$ & $\gamma$ \\
\hline$>-50 \mathrm{nT}$ & 3.3 & 0.2 \\
$-100 \mathrm{nT}<$ Dst $<-50 \mathrm{nT}$ & 3.1 & 0.19 \\
$-150 \mathrm{nT}<$ Dst $<-100 \mathrm{nT}$ & 3.5 & 0.25 \\
$<-150 \mathrm{nT}$ & 3.5 & 0.18 \\
\hline
\end{tabular}

Here, we assumed the Dst index decrease linearly to Dst ${ }_{\min }$

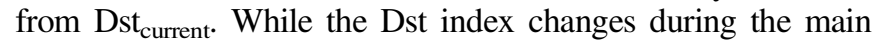
phase can be predicted using equation (11), the recovery phase's equation is required to be derived. Figure 4 is a superposed epoch plot of the Dst index for the storms' recovery phases listed in Tables A.1 and A.2 (Appendix). Here the minimum Dst is normalized to -1 . We examined several fitting functions to derive an equation describing the Dst index trends for the recovery phase. We found that an equation represented by two exponential functions, such as equation (12), effectively follows the recovery phase trend. Here, equation (12) presented by the red line is not the averaged Figure 4 but just a plausible expression derived by intuition. Other researchers can get a different equation.

$$
\begin{aligned}
& \operatorname{Dst}(t)_{\text {predict }}=-\left(\text { Dst }_{\text {current }}-\text { Dst }_{\text {min }}\right) \\
& \times\left(-0.41 \times \exp \left(-1 \times\left(t-T_{\text {min }}\right) \times \frac{1}{10.93}\right)\right. \\
&+(-0.41) \times \exp \left(-1 \times \frac{t-T_{\text {min }}}{13.96}\right) \\
&-0.179) \text { where } t=\left(T_{\text {min }}+1\right) \sim 24 .
\end{aligned}
$$

From equation (11), we can get the profile of Dst variation for the storm main phase and from equation (12) for the recovery phase from $1 \mathrm{~h}$ to $24 \mathrm{~h}$ ahead. During a storm is progressing, Dst $_{\text {min }}$ is updated every time, and if the predicted Dst $t_{\text {min }}$ is less than the current Dst, the empirical model recognizes the storm is in the recovery phase. Otherwise, if the Dst $t_{\min }$ is larger than the current Dst, then the algorithm predicts another storm main phase. Thus, this model can generate multi-peak storm prediction. These values are used as input parameters of the ANN model.

\section{Combination model}

The KASI Dst prediction (KDP) model effectively predicts the Dst index by combining the empirical and ANN models. First, we estimate the Dst index from $1 \mathrm{~h}$ to $24 \mathrm{~h}$ ahead using empirical equations (11) and (12). The prediction results from the empirical model are combined with the measurements listed in Table 1. For example, the 6th ANN model's input parameters are the $6 \mathrm{~h}$ ahead prediction value from equations (11) and (12) and the measurements in Table 1. Hence, it is as if there are 24 independent models for predicting Dst for each hour. Even if the predicted Dst from the empirical model is just one among

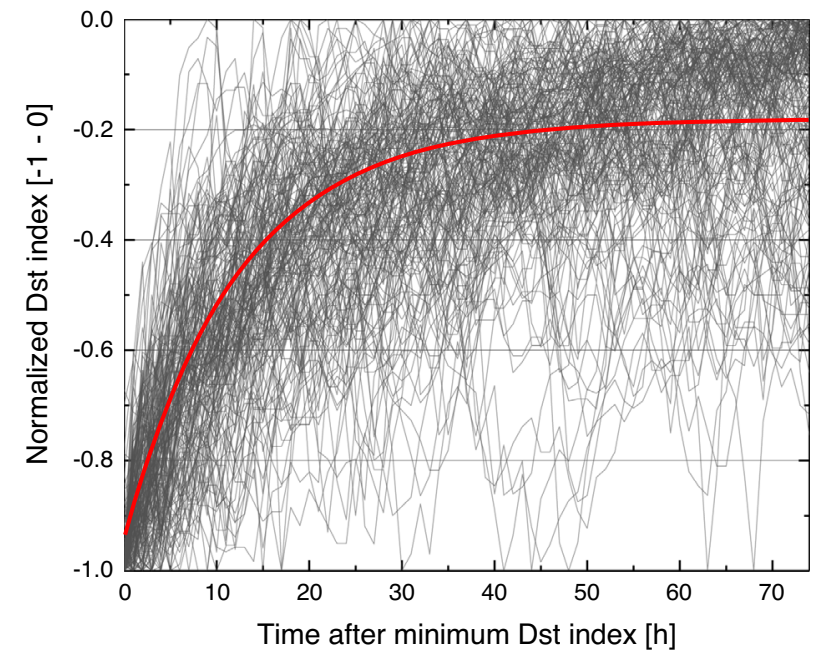

Fig. 4. The trend of the recovery phase of geomagnetic storms and results obtained from empirical equations. The Dst index is normalized by the absolute value of the minimum Dst index. Black solid lines show the normalized Dst index of the 184 events listed in Tables A.1 and A.2 in Appendix, and the red line is the result obtained from the recovery phase equation (12).

15 inputs, note ANN gives greater weight to the empirical predictions. Therefore, just adding the empirical model outputs on the ANN's input data improves the final prediction performance. The method of training and validation is the same as the original ANN model. Table 4 shows the combination model's hyperparameters.

Figure 5 shows an example of how the KDP model predicts the Dst index during geomagnetic storms. A halo CME was observed at 08:06 UT on 18 November 2003 by Solar and Heliospheric Observatory (SOHO) Large Angle and Spectrometric Coronagraph (LASCO) C2. At this time, the CME's speed was approximately $1150 \mathrm{~km} / \mathrm{s}$, and it would reach the Earth after approximately $32 \mathrm{~h}$ by simply dividing the Sun-Earth distance $(150,000,000 \mathrm{~km})$ by the solar wind speed $(1150 \mathrm{~km} / \mathrm{s})$. However, there were big uncertainties at this moment whether the geomagnetic storm would occur by this CME, how much the Dst index would decrease, how fast it would reach the minimum Dst, and how long it would take recovery. At this moment, space weather operators set the KDP as CME model and wait for the CME shock arrival. The ACE satellite observed the CME shock at 07:26 UT on 20 November, indicating that the arrival time was approximately $47 \mathrm{~h}$, approximately $15 \mathrm{~h}$ later than expected. However, the model did not predict geomagnetic storms because the IMF $B_{z}$ component was northward at the shock arrival. In the bottom panel of Figure 5, each color dots indicate the Dst values predicted $1 \mathrm{~h}$ at each hour, and the lines show the following predicted values for $24 \mathrm{~h}$ with one-hour interval. Note the first red dot indicates around zero, and no significant Dst changes are expected for $24 \mathrm{~h}$. As time goes on, the colored dots and lines show the magnetic storm proceeding.

As the IMF $B_{z}$ component rapidly turned southward at 10:45 UT on 20 November, the KDP model started to predict a minor storm event, where a Dst minimum of $-140 \mathrm{nT}$ would be reached around 13:00 UT. As the IMF enhanced southward 
Table 4. Optimized hyperparameters of ANN of combination models.

\begin{tabular}{lccc}
\hline & $\begin{array}{c}\text { Hidden layer } \\
\text { node number } \\
\text { (CME/CIR) }\end{array}$ & $\begin{array}{c}\text { Learning rate } \\
\text { (CME/CIR) }\end{array}$ & $\begin{array}{c}\text { Learning cycle } \\
\text { (CME/CIR) }\end{array}$ \\
\hline 1-hour prediction & $4 / 4$ & $0.01 / 0.01$ & $1500 / 1500$ \\
2-hour prediction & $24 / 55$ & $0.01 / 0.01$ & $1400 / 800$ \\
3-hour prediction & $5 / 58$ & $0.01 / 0.001$ & $1300 / 1500$ \\
4-hour prediction & $5 / 45$ & $0.01 / 0.001$ & $1300 / 1500$ \\
5-hour prediction & $6 / 43$ & $0.01 / 0.001$ & $1500 / 1400$ \\
6-hour prediction & $5 / 17$ & $0.01 / 0.001$ & $1300 / 1500$ \\
7-hour prediction & $2 / 24$ & $0.001 / 0.001$ & $1400 / 200$ \\
8-hour prediction & $2 / 25$ & $0.001 / 0.002$ & $1400 / 1200$ \\
9-hour prediction & $2 / 25$ & $0.001 / 0.003$ & $1400 / 1200$ \\
10-hour prediction & $2 / 25$ & $0.001 / 0.004$ & $1400 / 1200$ \\
11-hour prediction & $2 / 1$ & $0.001 / 0.0001$ & $1400 / 1100$ \\
12-hour prediction & $2 / 1$ & $0.001 / 0.0001$ & $1400 / 1100$ \\
13-hour prediction & $32 / 1$ & $0.0001 / 0.0001$ & $500 / 1100$ \\
14-hour prediction & $37 / 1$ & $0.0001 / 0.0001$ & $500 / 1100$ \\
15-hour prediction & $37 / 1$ & $0.0001 / 0.0001$ & $500 / 1100$ \\
16-hour prediction & $37 / 58$ & $0.0001 / 0.001$ & $500 / 1500$ \\
17-hour prediction & $37 / 60$ & $0.0001 / 0.001$ & $500 / 1500$ \\
18-hour prediction & $37 / 60$ & $0.0001 / 0.001$ & $500 / 1500$ \\
19-hour prediction & $37 / 1$ & $0.0001 / 0.0001$ & $500 / 1100$ \\
20-hour prediction & $37 / 1$ & $0.0001 / 0.0001$ & $500 / 1100$ \\
21-hour prediction & $37 / 1$ & $0.0001 / 0.0001$ & $500 / 1100$ \\
22-hour prediction & $37 / 1$ & $0.0001 / 0.0001$ & $500 / 1100$ \\
23-hour prediction & $37 / 1$ & $0.0001 / 0.0001$ & $500 / 1100$ \\
24-hour prediction & $37 / 1$ & $0.0001 / 0.0001$ & $500 / 1100$ \\
\hline
\end{tabular}

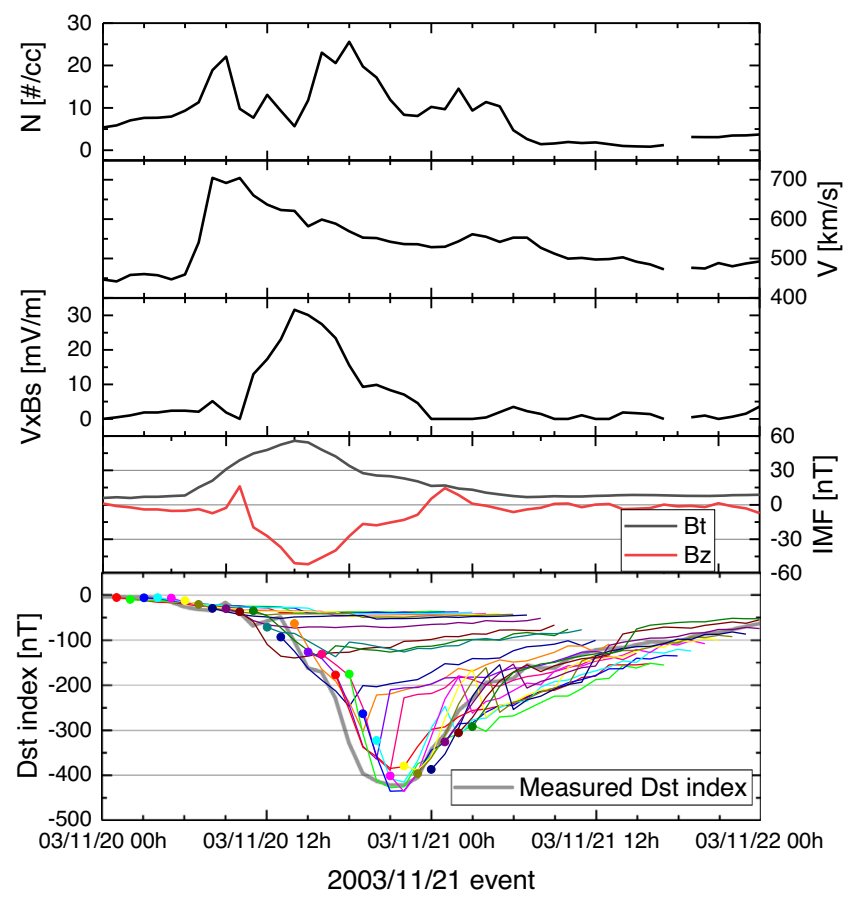

Fig. 5. Example of predicting the geomagnetic storm that occurred by CME on 20 November 2003. From top: (a) the solar wind density, (b) IMF total field, (c) IMF $B z$, (d) solar wind speed, $V \times B s$, and (e) Dst index measured and predicted. In the bottom panel, the gray line shows the measured Dst index, the color dots indicate the value predicted $1 \mathrm{~h}$ at each hour, and the colored lines show predicted Dst values for $24 \mathrm{~h}$ with a one-hour interval.

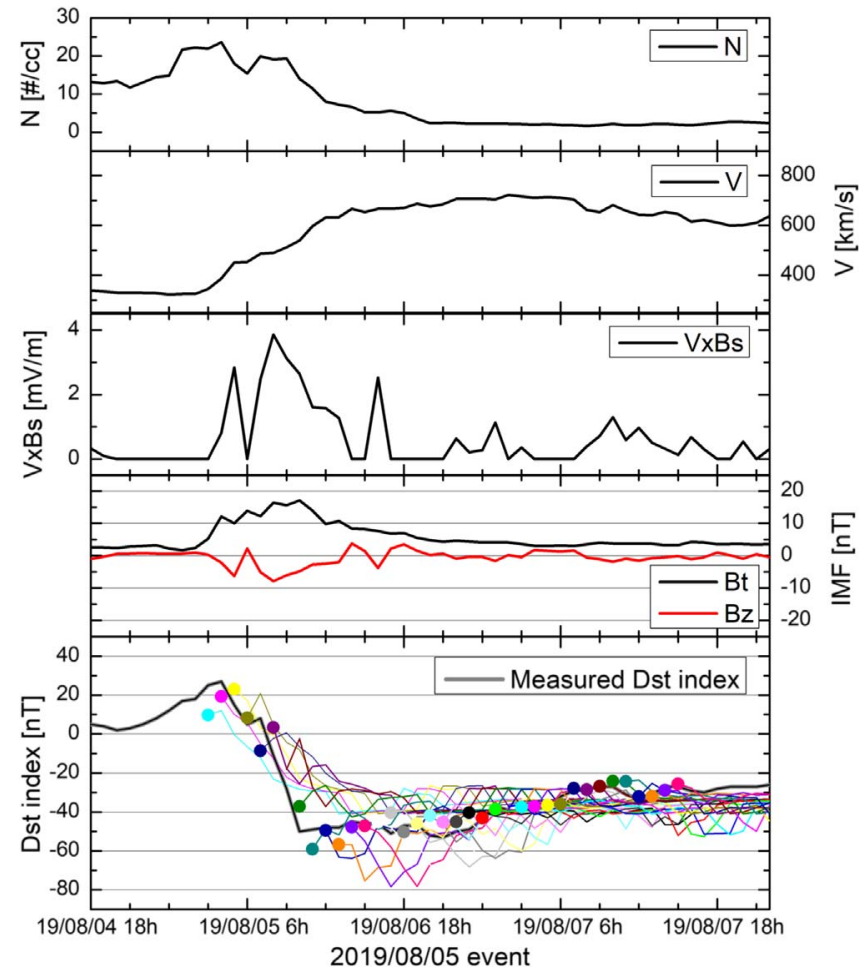

Fig. 6. Example of predicting the geomagnetic storm that occurred by CIR on 5 August 2019. Same format with Figure 5.

and the solar wind speed increased, the KDP model produced new predictions over time and provided results similar to actual Dst values. At 12:00 UT, the KDP model predicted that a Dst minimum of $-430 \mathrm{nT}$ would be reached around 20:00 UT. This value was similar to the measured value of $-422 \mathrm{nT}$ at 21:00 UT. At 21:00 UT on 20 November, the KDP model predicted that the storm would recover to $-100 \mathrm{nT}$ after one day. In this manner, space weather operators can deliver information about space weather conditions to users several hours before the minimum Dst is reached.

Figure 6 shows another example of KDP model prediction where the magnetic storm occurred by CIR on 5 August 2019. Generally, CIR could be identified by the corona holes on the solar UV images and produces minor magnetic storms. From 03:00 UT on 5 August, the solar wind speed increased slowly to about $700 \mathrm{~km} / \mathrm{s}$, similar speed to the CME storm shown in Figure 5. However, the IMF $B_{z}$ was larger than $-10 \mathrm{nT}$ during the storm was progressing and caused different scale storms from the CME-driven storm described in Figure 5. At the initial stage of the storm, the KDP model predicted the Dst index well. However, the solar wind speed increased extraordinary, and the model overestimates the minimum Dst with $-80 \mathrm{nT}$ while the measurement was larger than $-50 \mathrm{nT}$. Nevertheless, the model successfully predicted the minor storm caused by CIR.

Regarding the KDP model performance, Figure 7 shows the correlation coefficient and RMSE derived from equations (5) and (6) for the Dst index predicted using the empirical model, ANN, and combination model. Here, the statistical results are calculated only during the storm events listed in Tables A.1 and A.2 (Appendix) because we tried to develop the KDP 

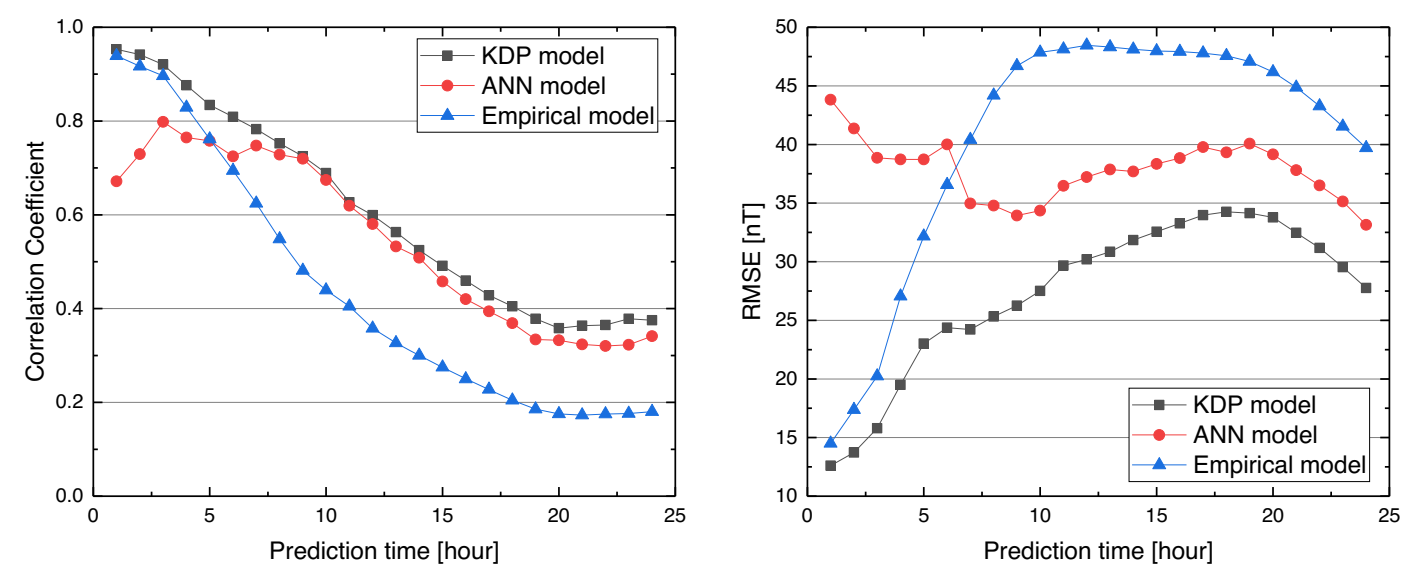

Fig. 7. Correlation coefficient and RMSE for the empirical model, ANN model, and combination (KDP) model. The performances were calculated only for the storm events in test group.
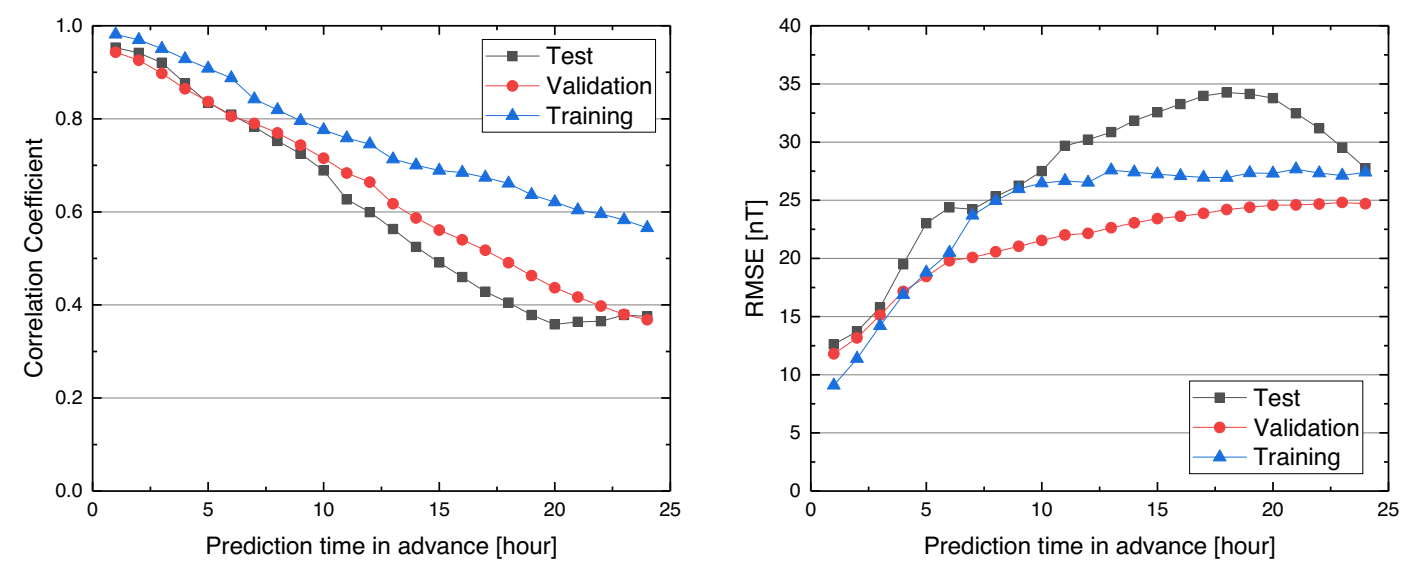

Fig. 8. KDP model's performance for training, validation, and test period.

model for space weather operation. In other literature, the space weather model's performance has been shown for both storm and quiet periods. These algorithm performances would be governed by the quiet period because it is considerably longer than the storm period. However, from a space weather operator's perspective, the important thing is how storm events are accurately predicted. We believe a better operational model should have good performance in predicting storm events. Thus, these results are obtained for the storm events in the test period. Figure 8 shows the KDP model's correlation coefficients and RMSEs for training, validation, and test group to illustrate the model's prediction capability. The higher correlation coefficient and smaller RMSE show that the Dst index is predicted more accurately using the combination model than the empirical and ANN models.

\section{Discussion and conclusion}

This paper introduces the KDP model that combined an ANN and an empirical model. The combination model showed better performance than each model in predicting the Dst index from $1 \mathrm{~h}$ to $24 \mathrm{~h}$ ahead, as shown in Figure 7. Therefore, we conclude that better space weather prediction can be achieved by using the combined models.

While this study aims to show an ANN model's improvement by combining an empirical model, as shown in Figure 7, there have been demands for comparing the KDP model's performance with others. While the ANN in the KDP model is trained only during the storm periods, we can find the input parameters even in quiet time. Thus, the KDP model can produce non-storm predictions. Figure 9 shows the storm and quiet time results, implying the combination model has superior prediction performance than other developed models. However, such a comparison does not tell us which model is better or worse because the test periods and conditions are different. The persistence model shown in Figure 9 assumes the prediction values are the same as the current Dst. Figure 9 clearly shows the KDP model's performance is better than the persistence model, and this model might be useful in space weather operation.

In addition to the persistence model, prediction efficiency is also used to validate the space weather model. Figure 10 shows the prediction efficiency of the KDP model calculated as below:

$$
\mathrm{PE}=1-\frac{\sum_{s=1}^{M}\left(\mathrm{Dst}_{s}-\mathrm{Dst}_{s}^{n n}\right)^{2}}{\sum_{s=1}^{M}\left(\mathrm{Dst}_{s}-\overline{\mathrm{Dst}}\right)^{2}}
$$



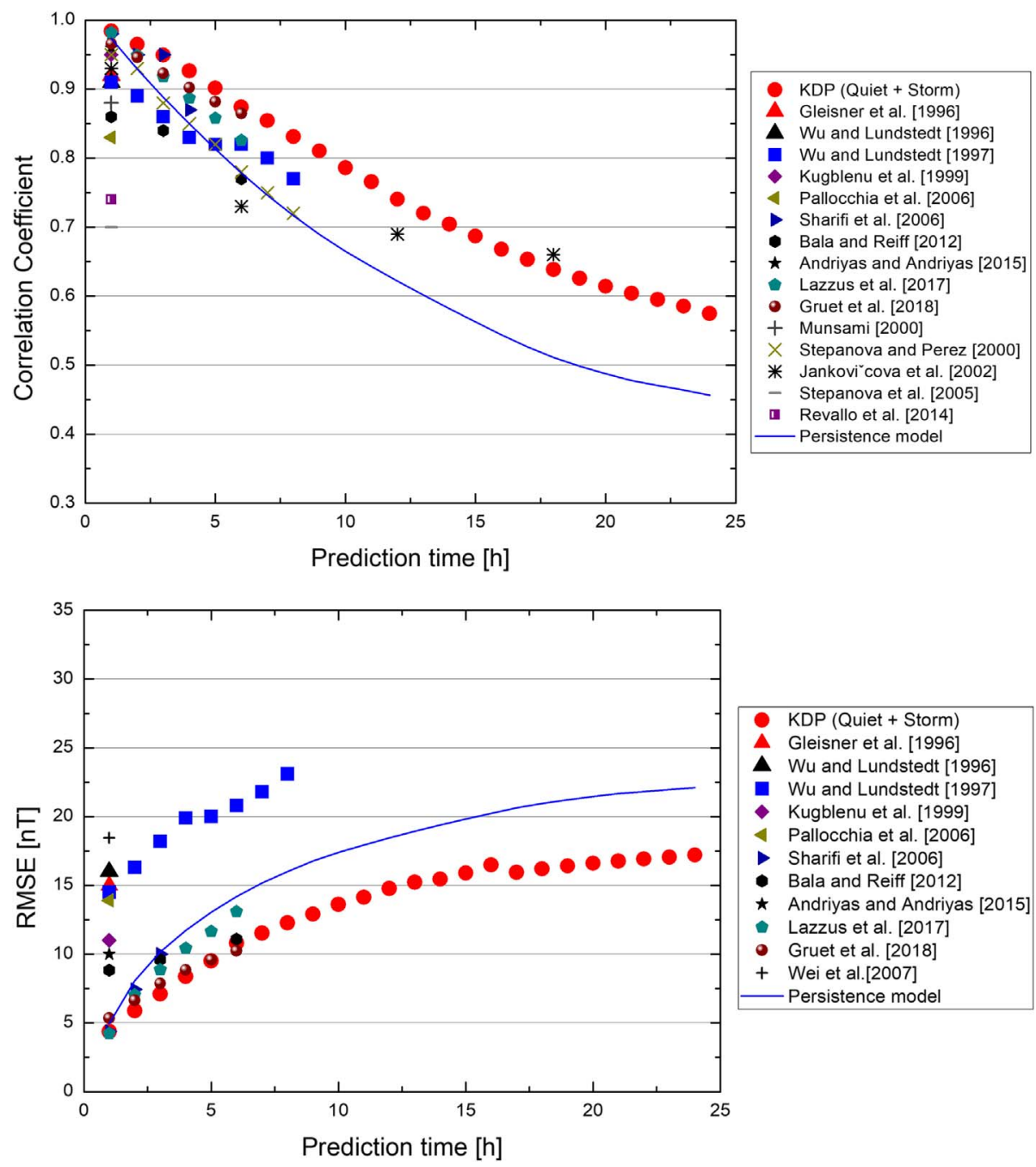

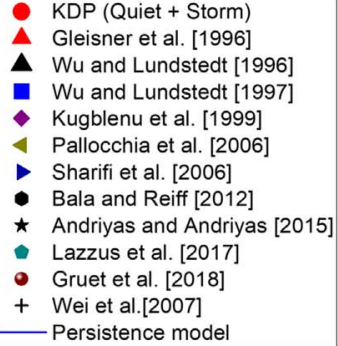

Fig. 9. Comparison of KDP model performance with other models: correlation coefficient (up) and RMSE (down). The red circles show prediction results from the KDP model for both quiet and storm time, and the red triangles indicate the model performance for only storm time.

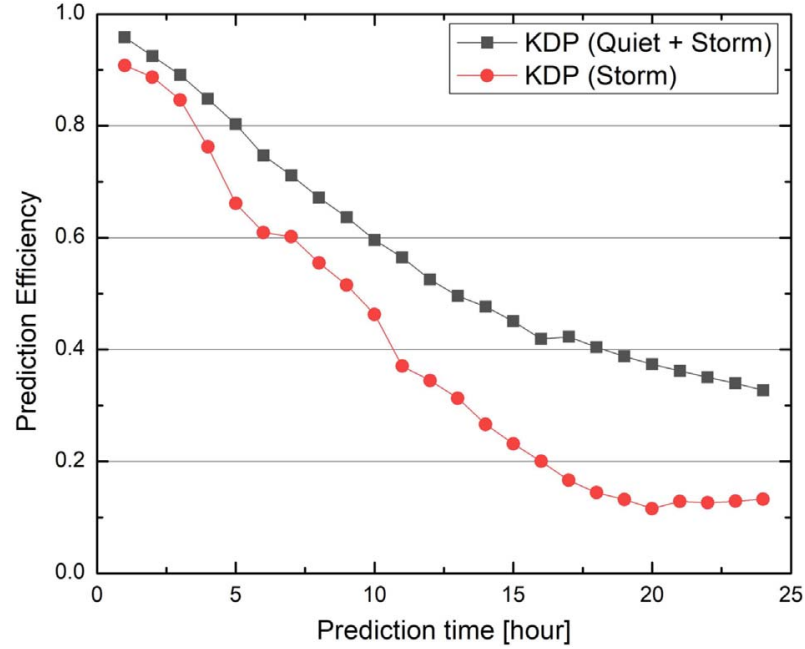

Fig. 10. Prediction efficiency of KDP model for storm time only (red) and all test period (black). where Dst , Dst $_{s}^{n n}$, and Dst stand for the measured, predicted, and mean Dst values respectively, and $M$ is the length of the record. The prediction efficiency tests the ability of the model to predict the variation of Dst around the mean. A prediction efficiency of 1 is perfect agreement at all times. Prediction efficiencies less than or equal to zero do not provide useful predictions of the observations' time variation. Figure 10 shows the prediction efficiency decrease as prediction time increases, while the model is still useful in predicting the 24-hour Dst index.

It should be noted that the ANN and empirical models used in this study are developed with a conventional method. The ANN is a commonly used feed-forward network, and roughly estimated equations implement the empirical models. As shown in Figure 2, the ANN model is not superior to other existing models. As shown in Figure 3, the minimum Dst values are spread widely, and the empirical equations are not fitted well to the data. Nevertheless, the combined model yielded better results, which means the combination model could be significantly improved by adopting modernized ANN and sophisticate 


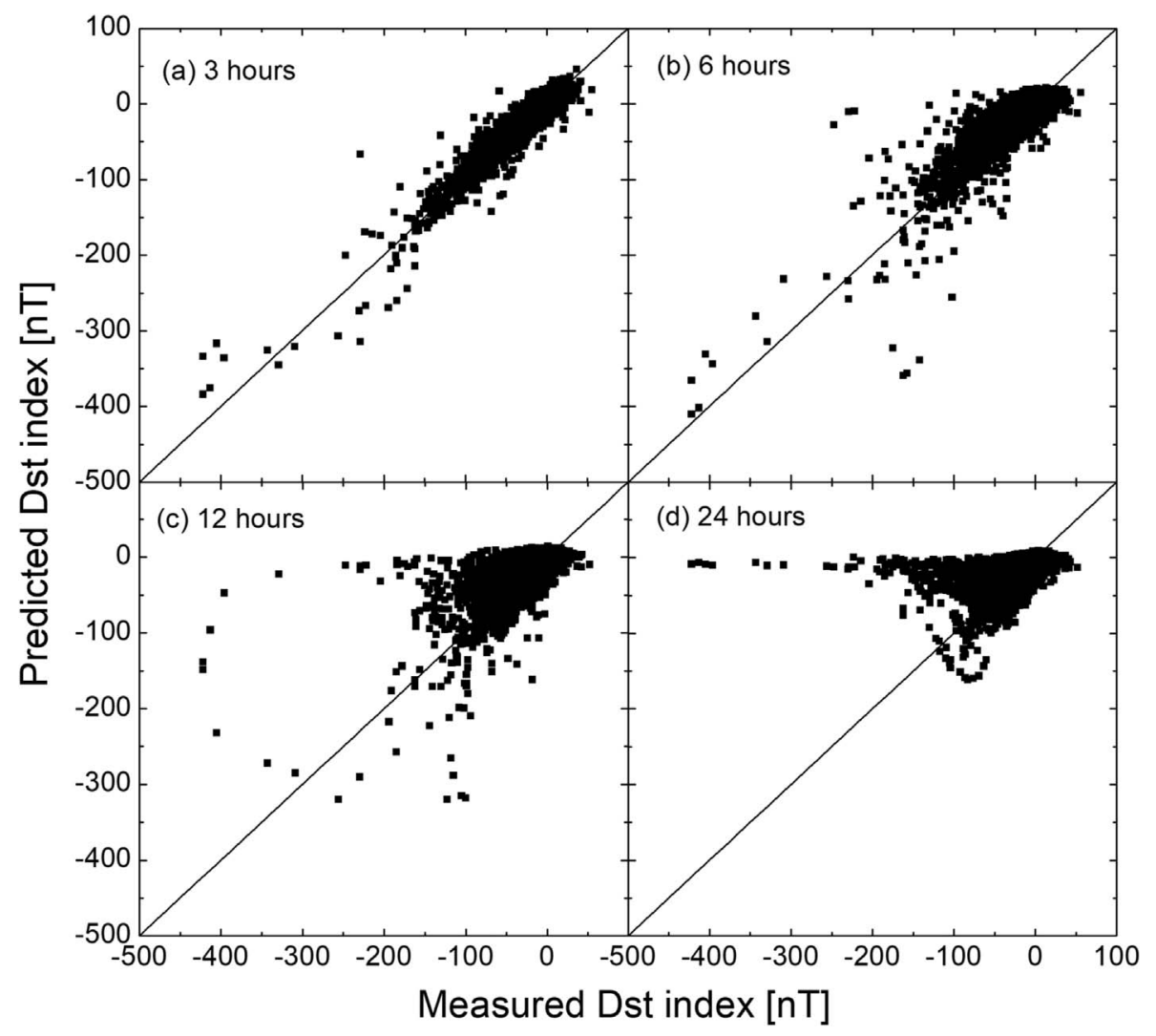

Fig. 11. Correlation between measured and predicted Dst index for (a) 3-hour (b) 6-hour, (c) 12-hour, and (d) 24-hour ahead.

empirical models. Further research will improve the empirical equations for predicting the Dst index. If two improved models are combined, the prediction accuracy would be more increase. Thus, this paper encourages researchers to improve their neural network models by adopting empirical models.

Besides, by categorizing geomagnetic storms into CMEdriven and CIR-driven storms, we improved the model performance. Through such categorization, we can increase the correlation between the Dst index and input parameters. In a future study, the solar cycle and seasonal effects could also be considered to make an improved empirical model. Space weather operators can easily recognize whether CME or CIR will cause magnetic storms from solar images. However, sometimes CMEs could be embedded in a CIR and resulting in a compound storm event (Al-Shakarchi \& Morgan, 2018). In this case, the decision for selecting CME or CIR mode remains to the operators. Even if they fail in identifying CME or CIRdriven storms, the KDP model predicts the Dst index anyhow by solar wind and current Dst.

The KDP model has been implemented to predict the Dst index $24 \mathrm{~h}$ ahead, and the prediction output is updated every hour for space weather operations. In general, space weather information is delivered to a professional group of users, such as radio communication companies, satellite operators, and flight dispatchers. Space weather users state that they can cope with severe space weather conditions in reasonable prediction time. For example, flight dispatchers (Privet communication with Korea air dispatchers) say they can change routes $3 \mathrm{~h}$ before a flight. It is well known that satellite launches can be delayed just a few minutes before launch. GEO satellite operators monitor their satellites for $24 \mathrm{~h}$, and they can act quickly for emergencies. As shown in Figure 7, the KDP model can predict the Dst storms $6 \mathrm{~h}$ ahead with a correlation coefficient of 0.8 and an RMSE of $24 \mathrm{nT}$ or less. Figure 11 shows the correlations between measurements and prediction values for 3-hour, 6-hour, 12-hour, and 24-hour ahead. The KDP model shows good correlations up to 6-hour ahead. We emphasize these are acceptable characteristics for space weather operation. Thus, this model can be used as an operational model for delivering warning signals to space weather users.

We have noted that space weather users do not want to know only the minimum Dst index but the recovery time of storms. Users like to know when they can return to their daily lives. Even though the 24-hour prediction correlation coefficient of the KDP model is not satisfactory at 0.38 , we attempted to develop a model to track the storm's progress using the 24-hour prediction model and predicted the recovery phase. While the Dst index's recovery does not mean that a space storm event has ended completely, it would be helpful information for space weather alerts.

Acknowledgements. The Dst index is obtained from NASA (https://omniweb.gsfc.nasa.gov/ow.html), and the hourly average solar wind data from ACE and DSCOVR are acquired from CDAWeb (https://cdaweb.gsfc.nasa.gov/index.html). For the model's operation, current Dst and solar wind data are 
downloaded from World Data Center for Geomagnetism, Kyoto (http://wdc.kugi.kyoto-u.ac.jp/dst_realtime/presentmonth/index. html) and NOAA (https://services.swpc.noaa.gov/products/ solar-wind/).

This work was supported by the National Meteorological Satellite Centre (NMSC) of the Korea Meteorological Administration (KMA) through the "Geostationary Meteorological Satellite Ground Segment Development" research project. The editor thanks two anonymous reviewers for their assistance in evaluating this paper.

\section{References}

Al-Shakarchi DA, Morgan H. 2018. Properties of the HPS-ICME-CIR interaction event of 9-10 September 2011. J Geophys Res (Space Phys) 123: 2535-2556. https://doi.org/10.1002/2017JA024849.

Bhaskar A, Vichare G. 2019. Forecasting of SYMH and ASYH indices for geomagnetic storms of solar cycle 24 including St. Patrick's day, 2015 storm using NARX neural network. J Space Weather Space Clim 9: A12. https://doi.org/10.1051/swsc/2019007.

Bingham ST, Mouikis CG, Kistler LM, Boyd AJ, Paulson K, Farrugia CJ, Huang CL, Spence HE, Claudepierre SG, Kletzing C. 2018. The outer radiation belt response to the storm time development of seed electrons and chorus wave activity during CME and CIR driven storms. J Geophys Res 123: 10139-10157. https://doi.org/10.1029/2018JA025963.

Birn J, Drake JF, Shay MA, Rogers BN, Denton RE, Hesse M, Kuznetsova M, Ma ZW, Bhattacharjee A, Otto A, Pritchett PL. 2001. Geospace environmental modeling (GEM) magnetic reconnection challenge. $J$ Geophys Res 106(A3): 3715-3719. https://doi.org/10.1029/1999JA900449.

Boyle CB, Reiff PH, Hairston MR. 1997. Empirical polar cap potentials. J Geophys Res 102(A1): 111-125. https://doi.org/ 10.1029/96JA01742.

Burton RK, McPherron RL, Russell CT. 1975. An empirical relationship between interplanetary conditions and Dst. J Geophys Res 80: 4204-4214. https://doi.org/10.1029/JA080i031p04204.

Daglis IA, Thorne RM, Baumjohann W, Orsini S. 1999. The terrestrial ring current: Origin, formation, and decay. Rev Geophys 37(4): 407-438. https://doi.org/10.1029/1999RG900009.

Eastwood J, Nakamura R, Turc L, Mejnertsen L, Hesse M. 2017. The scientific foundations of forecasting magnetospheric space weather. Space Sci Rev 212(3-4): 1221-1252. https://doi.org/ 10.1007/s11214-017-0399-8.

Echer E, Gonzalez WD, Guarnieri FL, Dal Lago A, Vieira LEA. 2005. Introduction to space weather. Adv Space Res 35: 855-865. https://doi.org/10.1016/j.asr.2005.02.098.

Freeman J, Nagai A. 1993. The Magnetospheric Specification and Forecast Model: Moving from real time to prediction. In: SolarTerrestrial Predictions IV, Proceeding of a Workshop at Ottawa, Canada, May 18-22, 1992, Vol 2, Hruska J, Shea MA, Smart DF, Heckman G, (Eds.), NOAA, Boulder. pp. 524-539.

Feldstein YI. 1992. Modelling of the magnetic field of magnetospheric ring current as a function of interplanetary medium parameters. Space Sci Rev 59(1-2): 83-165. https://doi.org/ 10.1007/BF01262538.

Gonzalez WD, Joselyn JA, Kamide Y, Kroehl HW, Rostoker G, Tsurutani BT, Vasyliunas VM. 1994. What is a geomagnetic storm? J Geophys Res 99(A4): 5771-5792. https://doi.org/10.1029/93JA02867.

Gardner M, Dorling S. 1998. Artificial neural networks (the multilayer perceptron) a review of applications in the atmospheric sciences. Atmos Environ 32(14): 2627-2636. https://doi.org/ 10.1016/S1352-2310(97)00447-0.

Gruet MA, Chandorkar M, Sicard A, Camporeale E. 2018. Multiplehour-ahead forecast of the Dst index using a combination of long short-term memory neural network and gaussian process. Space Weather 16: 1882-1896. https://doi.org/10.1029/2018SW001898.

Haykin S. 1998. Neural Networks: A Comprehensive Foundation. Prentice Hall, Upper Saddle River, NJ. ISBN 0132733501.

Kugblenu S, Taguchi S, Okuzawa T. 1999. Prediction of the geomagnetic storm associated Dst index using an artificial neural network algorithm. Earth Planet Space 51: 307-313. https://doi. org/10.1186/BF03352234.

Lazzús JA, Vega P, Rojas P, Salfate I. 2017. Forecasting the Dst index using a swarm-optimized neural network. Space Weather 15: 1068-1089. https://doi.org/10.1002/2017SW001608.

Lyons L. 1998. The geospace modeling program grand challenge. $J$ Geophys Res 103(A7): 14781-14785. https://doi.org/10.1029/ 98JA00015.

Lundstedt H, Wintoft P. 1994. Prediction of geomagnetic storms from solar wind data with the use of a neural network. Ann Geophys 12: 19. https://doi.org/10.1007/s00585-994-0019-2.

Lundstedt H, Gleisner H, Wintoft P. 2002. Operational forecasts of the geomagnetic Dst index. Geophys Res Lett 29: 2181. https://doi. org/10.1029/2002GL016151.

Liemohn MW, Jazowski M, Kozyra JU, Ganushkina N, Thomsen MF, Borovsky JE. 2010. CIR versus CME drivers of the ring current during intense magnetic storms. Proc $R$ Soc London A 466 (2123): 3305-3328. https://doi.org/10.1098/rspa.2010.0075.

Matamba TM, Habarulema JB. 2018. Ionospheric responses to CMEand CIR-driven geomagnetic storms along $30 \mathrm{E}-40 \mathrm{E}$ over the African sector from 2001 to 2015. Space Weather 16: 538-556. https://doi.org/10.1029/2017SW001754.

Makarov GA. 2018. Heliolatitude regularities of magnetically disturbed days with daily average geomagnetic index Dst $<-100 \mathrm{nT}$. Sol Terr Phys 4(3): 20-23. https://doi.org/10.12737/stp-43201803.

McPherron RL, O’Brien TP. 2001. Predicting geomagnetic activity: The Dst index. In: Space Weather Geophys Monogr Ser, vol. 125. Song P, Singer HJ, Siscoe GL (Eds.), AGU, Washington, DC. pp. 339-345. https://doi.org/10.1029/GM125p0339

Murayama T. 1982. Coupling function between solar wind parameters and geomagnetic indices. Reviews of Geophysics 20(3): 623-629. https://doi.org/10.1029/RG020i003p00623.

O'Brien TP, McPherron RL. 2000. Forecasting the ring current index Dst in real time. J Atmos Sol Terr Phys 62: 1295-1299. https://doi. org/10.1016/S1364-6826(00)00072-9.

Ohtani S-I, Fujii R, Hesse M, Lysak RL. 2000. Magnetospheric current systems, vol 118. American Geophysical Union, Washington, DC. ISBN 9781118669006.

Raeder J, Maynard N. 2001. Foreword (to Special Section on Proton Precipitation Into the Atmosphere). J Geophys Res 106(A1): 345 348. https://doi.org/10.1029/2000JA000600.

Rangarajan G. 1989. Indices of geomagnetic activity. Geomagnetism 3: $323-384$.

Rastätter L, Kuznetsova MM, Glocer A, Welling D, Meng X, Raeder J, et al. 2013. Geospace environment modeling 2008-2009 challenge: Dst index. Space Weather 11(4): 187-205. https://doi. org/10.1002/swe.20036.

Revallo M, Valach F, Hejda P, Bochníček J. 2014. A neural network Dst index model driven by input time histories of the solar windmagnetosphere interaction. J Atmos Sol Terr Phys 110-111: 9-14. https://doi.org/10.1016/j.jastp.2014.01.011.

Revallo M, Valach F, Hejda P, Bochnicek J. 2015. Modeling of CME and CIR driven geomagnetic storms by means of artificial neural 
networks. Contrib Geophys Geodesy 45(1): 53-65. https://doi.org/ 10.1515/congeo-2015-0013.

Romashets EP, Poedts S, Vandas M. 2008. Modeling of the magnetic field in the magnetosheath region. J Geophys Res (Space Phys) 113: A02203. https://doi.org/10.1029/2006JA012072.

Richardson IG, Cane HV. 2010. Near-earth interplanetary coronal mass ejections during solar cycle 23 (1996-2009): Catalog and summary of properties. Solar Phys 264: 189-237. https://doi.org/ 10.1007/s11207-010-9568-.

Shen XC, Hudson MK, Jaynes A, Shi Q, Tian A, Claudepierre S, et al. 2017. Statistical study of the storm time radiation belt evolution during Van Allen Probes era: CME- versus CIR-driven storms. J Geophys Res (Space Phys) 122: 8327-8339. https://doi. org/10.1002/2017JA024100.

Stepanova M, Antonova E, Troshichev O. 2005. Prediction of Dst variations from polar cap indices using time-delay neural network. J Atmos Sol Terr Phys 67: 1658-1664. https://doi.org/10.1016/ j.jastp.2005.02.027.

Sugiura M. 1964. Hourly values of equatorial Dst for the IGY. Ann Int Geophys Year 35: 7-45.

Temerin M, Li X. 2002. A new model for the prediction of Dst on the basis of the solar wind. J Geophys Res 107(A12): 1472. https://doi. org/10.1029/2001JA007532.

Temerin M, Li X. 2006. Dst model for 1995-2002. J Geophys Res 111: A04221. https://doi.org/10.1029/2005JA011257.
Wang CB, Chao JK, Lin C-H. 2003. Influence of the solar wind dynamic pressure on the decay and injection of the ring current. J Geophys Res 108: 1341. https://doi.org/10.1029/2003JA009851.

Wanliss JA, Showalter KM. 2006. High-resolution global storm index: Dst versus SYM-H. J Geophys Res 111(A2). https://doi.org/ 10.1029/2005JA011034.

Watanabe S, Sagawa E, Ohtaka K, Shimazu H. 2002. Prediction of the Dst index from solar wind parameters by a neural network method. Earth Planet Space 54: 1263-1275. https://doi.org/ 10.1186/BF03352454.

Watari S. 2017. Geomagnetic storms of cycle 24 and their solar sources. Earth Planet Space 69(1): 70. https://doi.org/10.1186/ s40623-017-0653-z.

Weigel RS. 2010. Solar wind density influence on geomagnetic storm intensity. J Geophys Res 115(A9). https://doi.org/10.1029/ 2009JA015062.

Wu J-G, Lundstedt H. 1996. Prediction of geomagnetic storms from solar wind data using Elman recurrent neural networks. Geophys Res Lett 23: 319-322. https://doi.org/10.1029/96GL00259.

Wu J-G, Lundstedt H. 1997. Geomagnetic storm predictions from solar wind data with the use of dynamic neural networks. J Geophys Res 102: 14255-14268. https://doi.org/10.1029/97JA00975. 


\section{Appendix}

Table A.1. CME-driven geomagnetic storm events.

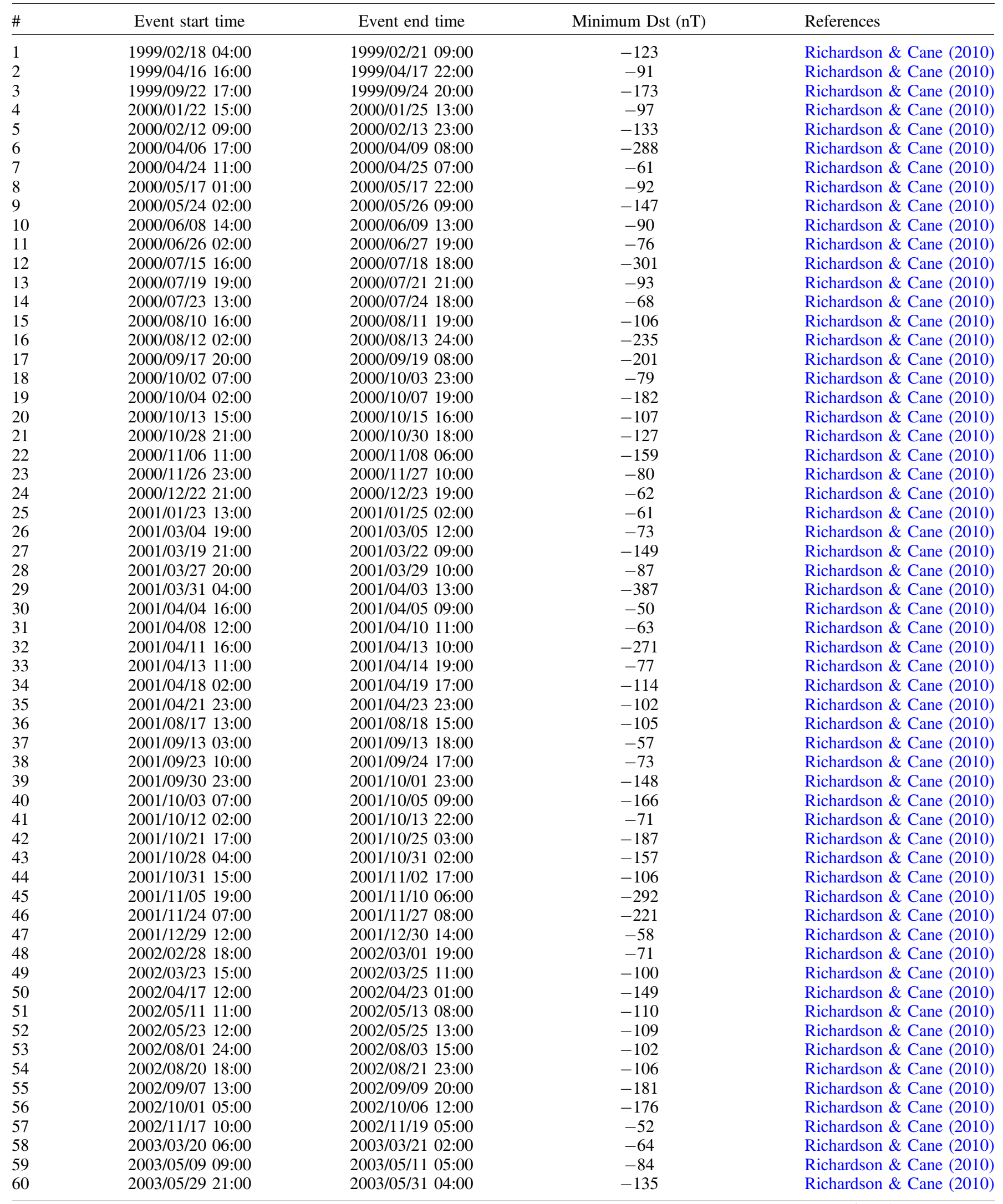


W. Park et al.: J. Space Weather Space Clim. 2021, 11, 38

Table A.1. (Continued)

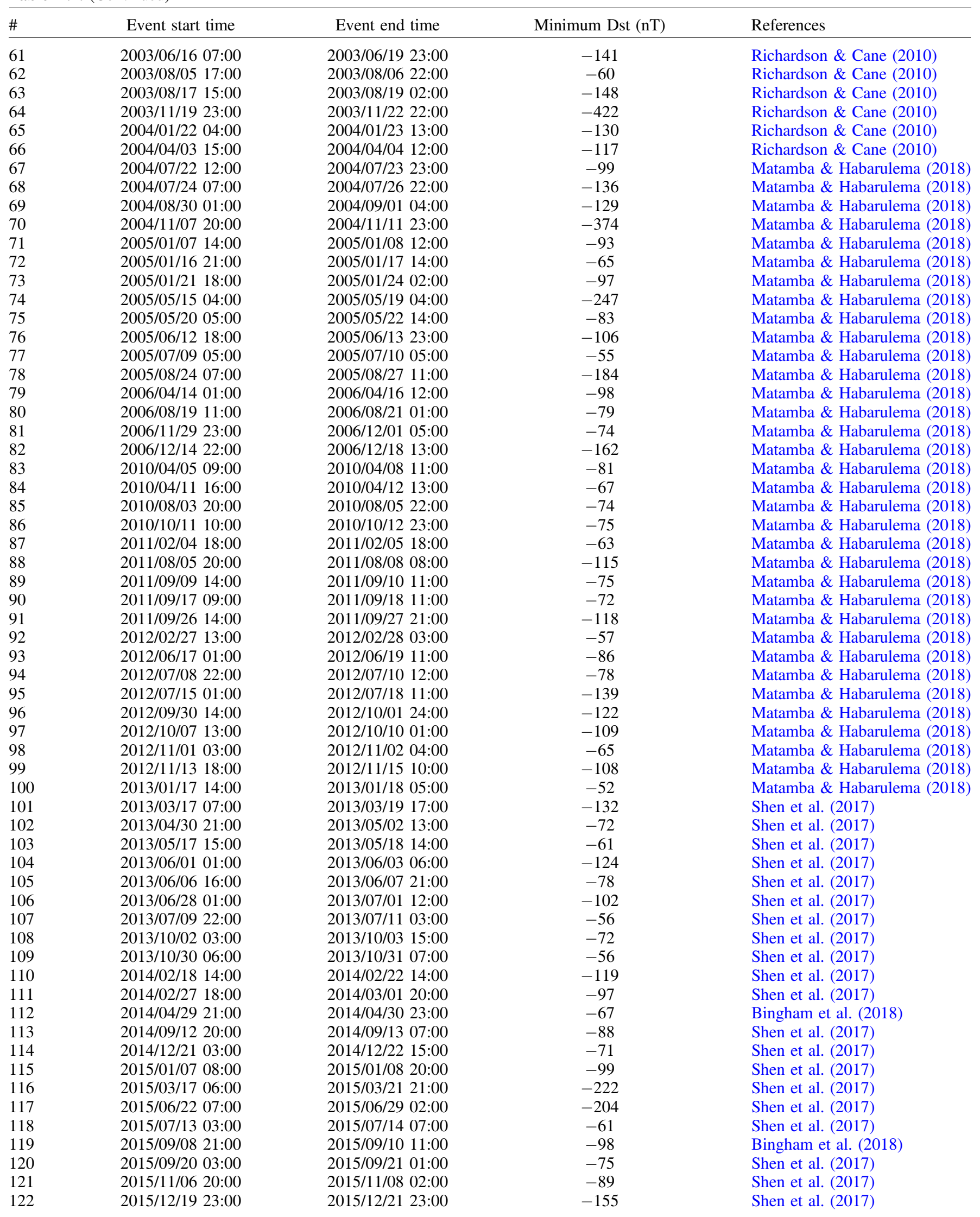


Table A.1. (Continued)

\begin{tabular}{lcccl}
\hline$\#$ & Event start time & Event end time & Minimum Dst (nT) & References \\
\hline 123 & $2015 / 12 / 31 ~ 12: 00$ & $2016 / 01 / 0121: 00$ & -110 & Shen et al. (2017) \\
124 & $2016 / 10 / 13$ 03:00 & $2016 / 10 / 14$ 14:00 & -104 & http://www.srl.caltech.edu/ACE/ASC/DATA/level3/icmetable2.htm \\
125 & $2017 / 05 / 27 ~ 22: 00$ & $2017 / 05 / 28 ~ 23: 00$ & -125 & http://www.srl.caltech.edu/ACE/ASC/DATA/level3/icmetable2.htm \\
126 & $2017 / 07 / 16 ~ 07: 00$ & $2017 / 07 / 1802: 00$ & -72 & http://www.srl.caltech.edu/ACE/ASC/DATA/level3/icmetable2.htm \\
127 & $2017 / 09 / 07 ~ 21: 00$ & $2017 / 09 / 1004: 00$ & -124 & http://www.srl.caltech.edu/ACE/ASC/DATA/level3/icmetable2.htm \\
\hline
\end{tabular}

Table A.2. CIR-driven geomagnetic storm events.

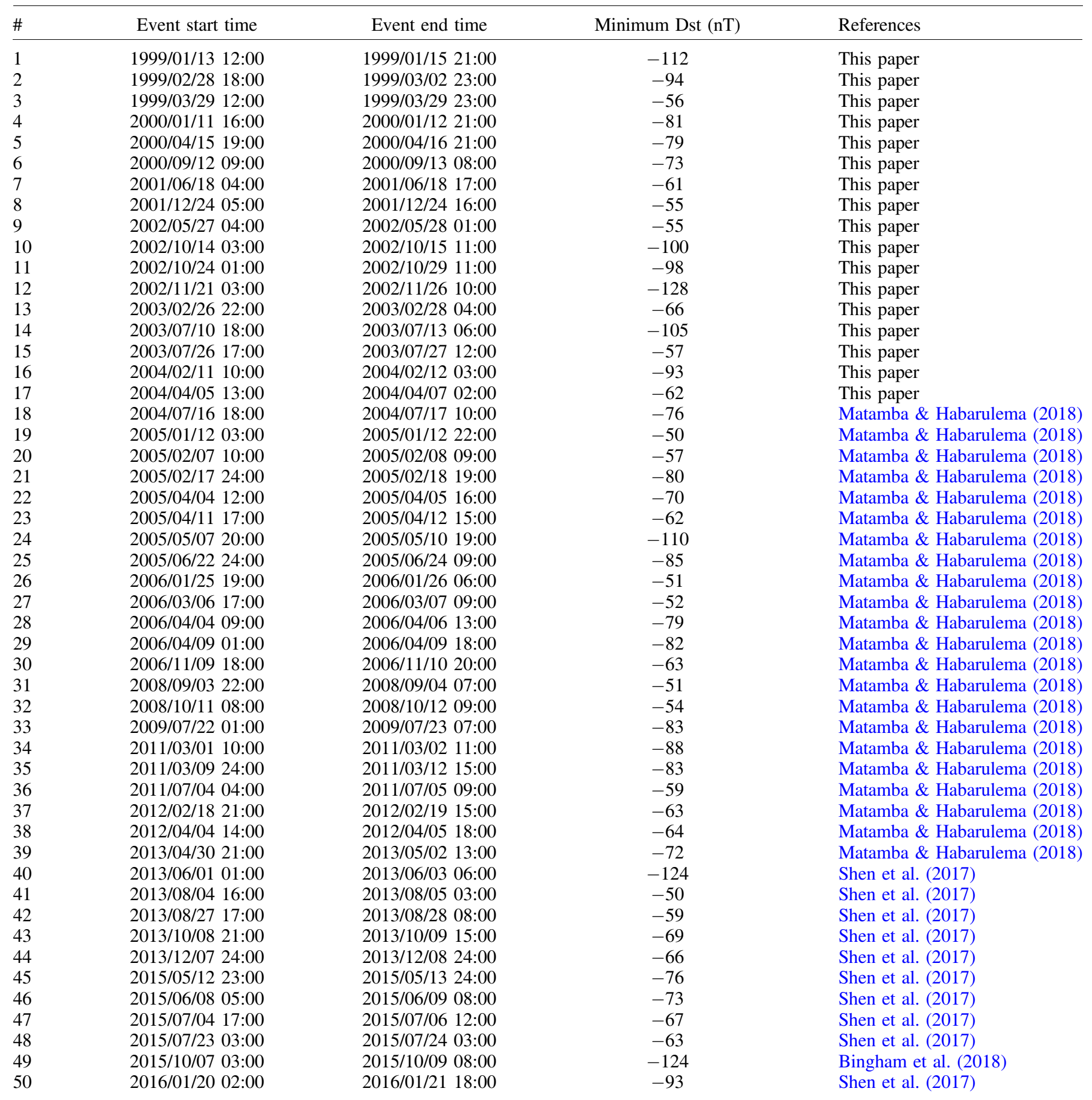


W. Park et al.: J. Space Weather Space Clim. 2021, 11, 38

Table A.2. (Continued)

\begin{tabular}{|c|c|c|c|c|}
\hline \# & Event start time & Event end time & Minimum Dst (nT) & References \\
\hline 52 & 2016/03/06 15:00 & 2016/03/08 09:00 & -98 & Shen et al. (2017) \\
\hline 54 & 2016/04/12 20:00 & 2016/04/13 09:00 & -55 & Shen et al. (2017) \\
\hline 55 & 2016/05/08 02:00 & $2016 / 05 / 10 \quad 12: 00$ & -88 & Shen et al. (2017) \\
\hline 56 & 2016/11/10 10:00 & 2016/11/11 03:00 & -59 & This paper \\
\hline 57 & $2017 / 03 / 2623: 00$ & $2017 / 03 / 28 \quad 11: 00$ & -74 & This paper \\
\hline
\end{tabular}

Cite this article as: Park W, Lee J, Kim K-C Lee J, Park K, et al. 2021. Operational Dst index prediction model based on combination of artificial neural network and empirical model. J. Space Weather Space Clim. 11, 38. https://doi.org/10.1051/swsc/2021021. 